\title{
ENTRE EL LENGUAJE DE MICROSOFT Y EL DE BORGES ${ }^{1}$
}

\author{
BETWEEN THE LANGUAGE OF MICROSOFT AND BORGES
}

\author{
LeOnel Sebastián Chavarría \\ Catedrático de la Universidad Nacional Autónoma de México
}

[Recepción: Agosto de 2010/ Conformidad: Setiembre de 2010]

\section{RESUMEN}

En el año 2008, el sindicato de trabajadores de la educación de la República Mexicana, sometió a consideración la siguiente pregunta: "¿Considera usted que la educación debe tener una mayor vinculación con el empleo y el aparato productivo?”. Esta encuesta presentó los siguientes resultados: Un contundente $92 \%$ respondió de manera afirmativa, el 1\% dijo no saber, y tan sólo el $7 \%$ ha dicho que no debe existir mayor vinculación. ¿Cuál es la opinión de usted?

Para responder esta pregunta, si se reflexiona un poco, primero debemos tener claro el nivel educativo al que se hace referencia con el término "educación" en dicho cuestionamiento. Si la encuesta se refiere a la educación superior o capacitación para el trabajo, la respuesta, en principio, considero que debería ser afirmativa.

En términos generales es fácil percibir la conveniencia de vincular a los estudiantes de estos niveles educativos con el mercado laboral para contribuir a enriquecer su naciente experiencia profesional, desarrollar ciertas habilidades profesionales $y$, de esta forma, aproximarlos a la posibilidad de obtener un anhelado empleo al término de sus estudios. Y más aún, bajo una estrategia visionaria dirigida a los estratos superiores del conocimiento, sería deseable que algunos institutos de investigación de nuestras universidades

\begin{abstract}
In 2008, the union representing education workers in Mexico, submitted for consideration the following question: "Do you think that education should be a strong link with employment and productive?". This survey showed the following results: a resounding $92 \%$ answered yes, $1 \%$ were unsure, and only $7 \%$ said that there should be greater linkage. What is your opinion?

To answer this question, if we reflect a bit, we must first be clear about the level of education referred to the term "education" in that question. If the survey relates to higher education or job training, response, in principle, I believe there should be affirmative.

In general terms it is easy to see the desirability of linking students from these levels of education to the labor market to help enrich their nascent professional experience, develop certain professional skills, and thus bring them closer to the possibility of obtaining a desired job to completion of their studies. And yet, in a visionary strategy aimed at the upper layers of knowledge, it is desirable that some research institutes in our universities to become the engine of development of various productive activities, using the example of Stanford University has been largely responsible for the impressive economic and technological development in the region of Silicon Valley.
\end{abstract}

1 Adaptación para Quipukamayoc y actualización para América Latina del artículo original publicado en dos partes en la Revista “Educación 2001", No. 155 de abril y No. 156 de mayo de 2008. México. (ISSN 1405-2075).

* Catedrático de tiempo completo, Secretario General (Vicedecano) de la Facultad de Contaduría y Administración de la Universidad Nacional Autónoma de México (UNAM) y Consejero Honorario de la Asociación Latinoamericana de Facultades y Escuelas de Contaduría y Administración (ALAFEC).1sebastian@correo.fca.unam.mx 
se conviertan en el motor de desarrollo de diversas actividades productivas, tomando como ejemplo a la Universidad de Stanford que ha sido en buena parte responsable del impresionante desarrollo tecnológico y económico de la región del Silicon Valley.

Sin embargo, es preciso comprender que quien formula la pregunta es el sindicato que tiene bajo su responsabilidad la educación básica en México. Por esta razón, podríamos asumir que el término "educación", en la encuesta, hace referencia a la enseñanza que se imparte en el nivel de estudios correspondientes al nivel primario. Bajo esta suposición y con el fin de precisar el nivel educativo al que se hace referencia, la pregunta inicial podría adoptar la siguiente forma: “ $i$ Considera usted que la educación básica y media superior deben tener una mayor vinculación con el empleo y el aparato productivo?”. De esta forma el cuestionamiento resulta extraño, ¿cómo vincular a estudiantes menores de edad con el sector laboral? Obviamente no es lógica la idea de una vinculación directa a través de prácticas laborales. Si no es así, ¿a qué obedece la encuesta que hace el sindicato más numeroso de América Latina?

Palabras clave: Educación y sistema productivo, desarrollo tecnológico y económico, Actividades productivas, educación y mercado laboral.

La intención de vincular a la educación con el mercado laboral obedece a una corriente utilitaria de la educación, en la que se ha reflexionado muy poco sobre su conveniencia, estrategias de vinculación, e importantes consecuencias. Esta corriente educativa es bien vista por un amplio sector de la sociedad, tal como lo demuestran los resultados de la encuesta. La contundente aceptación de esta tendencia en la educación incrementa el riesgo de influir el modelo educativo de los niveles de educación básica y media superior, provocando que una cultura utilitaria desplace a un lugar sin importancia a la enseñanza del conocimiento "que ya no se usa en el trabajo”. ¿Cómo lograr este objetivo desde
However, you must understand that asks the question who is the union that is responsible for basic education in Mexico. For this reason we may assume that the term "education" in the survey, refers to the education provided in educational attainment for the primary level. Under this assumption and to clarify the level of education referred to the initial question might take the following form: Do you think that the basic and upper secondary education must have a strong link with employment and productive? . Thus, the question seems strange. How to link to underage students with labor? Obviously the idea is not logical for a direct link through labor practices. If not, does that render the survey made by the largest union in Latin America?

Key words: education and productive system, technological and economic development, productive activities, education and labor market.

los niveles básicos de la educación?, ¿privilegiando la enseñanza de conocimientos de aplicación inmediata en el trabajo en lugar de estudiar las poco entendidas teorías científicas?, ¿dedicando más tiempo a la enseñanza de Excel que a la comprensión de problemas matemáticos resueltos con un anticuado lápiz?, ¿privilegiando más las clases de Internet que las de historia?, ¿despertando más el interés por las nuevas tecnologías que por la literatura?

En noviembre de 2004, en una reunión del Consejo Ejecutivo de la Asociación Latinoamericana de Facultades y Escuelas de Contaduría y Administración (ALAFEC) celebrada en Buenos Aires, Argentina, a la 
que fueron convocadas las autoridades de las instituciones de educación superior más importantes de América Latina y el Caribe en esas áreas pragmáticas del conocimiento, tuve la oportunidad de conocer al Doctor Jaim Etcheverry, entonces rector de la Universidad de Buenos Aires. En dicha reunión el Doctor Jaim realizó una breve e interesante reflexión en la que asocia a la educación utilitaria como parte de una tetralogía trágica para el sistema educativo de una nación. Fueron tan serios los argumentos que planteó ante un auditorio compuesto por una élite académica dedicada a formar hombres de empresa que me llevaron a estudiar su obra bibliográfica de la cual extraigo los siguientes textos que nos ayudaran a entender el fondo del problema que implica la adopción de una moda educativa orientada a formar al personal que supuestamente requieren las empresas y no a los ciudadanos que verdaderamente demanda el país Jaim afirma que:

"La preocupación central de nuestra sociedad es que lo que aprendan los jóvenes les "sirva”. Y pronto. Pero lo que "sirve" está, cada vez más, relacionado con la vida profesional, con el beneficio económico. [Se está generalizando el convencimiento] de que la principal función de la educación es preparar a los jóvenes para el trabajo [y no para la vida]. [Es] altamente preocupante la tendencia a la especialización precoz de los jóvenes, durante su educación media e, inclusive al comienzo de la superior [en lugar de esto] lo que hoy debería buscarse es generalizar precozmente, es decir, abrir el panorama vital de los jóvenes. Se desprestigia aceleradamente lo que, en términos generales, padres y alumnos juzgan "inútil”. Es decir, "inútil” para hacer dinero [...] El peligro es que la educación se convierta, insensiblemente, en una forma de ganarse la vida $y$ que deje de ser una experiencia que permita explorar el modo de vivir mejor [ $\mathrm{La}$ tragedia educativa sería convertir a la escuela en] un taller de entrenamiento de la fuerza laboral enseñando lo "útil”, a menudo para evitar que los jóvenes se formulen preguntas más profundas sobre la forma en que vivimos".

Si sumado a lo anterior, recordamos que con nuestra lamentable realidad social no son pocos los casos en los que nuestros niños y adolescentes, por necesidad económica, ya se ven obligados a vincularse con el trabajo poniendo en riesgo la conclusión de su formación escolar, ahora resulta que se pretende idear una estrategia para acercar a más educandos a las fábricas y empresas, y distanciarlos de las aulas de estudio o laboratorios de investigación. Qué paradoja, ¿qué no debería ser todo lo contrario?

El título de este artículo, que mezcla y parafrasea las ideas originales de Stoll y Jaim, trata de confrontarnos con la situación educativa que hoy vivimos y que se acentuará más con la irreflexiva aceptación de una educación supuestamente moderna y supuestamente útil para el desarrollo nacional.

A continuación, presento cinco argumentos a través de los cuales trato de sostener una postura contraria a la vinculación de la educación básica y media superior con el empleo, debido al daño intelectual que podemos hacer a la comunidad estudiantil de esos niveles educativos en cualquier nación Latinoamericana.

\section{LOS CONOCIMIENTOS FUNDAMENTALES PASAN A SEGUNDO TÉRMINO: CONCENTRADOS EN LO SECUNDARIO}

El problema no radica, per se, en enseñar conocimientos que se apliquen en la vida profesional, indiscutiblemente esa capacitación 
llega a ser importante, necesaria ó indispensable de acuerdo con el nivel educativo de que se trate. El problema radica en privilegiar los conocimientos de moda y relegar a un sitio carente de importancia a los conocimientos que son fundamentales para la formación integral de una persona.

En nuestra sociedad aumenta cada día más la idea de privilegiar la enseñanza de lo que se considera tecnológicamente moderno y útil para el trabajo, llevando a asumir superficialmente la temática educativa que se considera anticuada o inútil. En el fondo, esto es debido a que son muchas las personas, incluyendo a padres de familia, maestros y alumnos, que no encuentran respuesta a la siguiente pregunta: ¿Hoy en día para que sirve la historia, literatura, las artes, las ciencias o el análisis de problemas matemáticos? Al no encontrar respuesta, aceptan felizmente los conocimientos que estén de moda, sin mayor reflexión, sin caer en cuenta que para poder edificar algo, lo que sea, es necesario contar con sólidas bases o, de lo contrario, todo lo que se construya estará endeble.

Los educadores no pueden olvidar que los conocimientos sólidos sobre matemáticas, lengua y literatura, historia, artes y ciencias naturales, son las piedras angulares en la formación humana, y constituyen los cimientos que requiere toda persona que aspire seriamente a un alto desarrollo intelectual y no solo a ser un eficiente usuario de herramientas tecnológicas. Como dato importante debemos recordar que los concursos internacionales de evaluación educativa fijan su atención en temas relativos a las matemáticas, lectura y redacción, y no en el uso de software.

Frecuentemente he escuchado decir: "Esforzarte en solucionar un problema matemático sin el uso de las modernas tecnologías sería anticuado, éstas ya te dan las respuestas casi de manera inmediata”. Es necesario hacer ver a los educandos que estudiar sólo lo fácil, los llevará a ser dependientes intelectuales de quienes se atrevieron a estudiar y comprender lo difícil, lo cual es muy riesgoso para el desarrollo de una persona y de una sociedad.

\section{FORMAR HOMBRES DE NEGOCIOS: LO ÚTIL DE LA EDUCACIÓN “INÚTIL”}

Prepararse para ser hombre de negocios o alto directivo de una empresa es una moda profesional que está marcando el rumbo en la educación de los jóvenes de nuestro suelo y de nuestro tiempo. Pues bien, quienes compartan este anhelo deben saber que las universidades más prestigiadas del mundo tienen establecido como examen de ingreso a sus posgrados en negocios el GMAT (por las siglas en inglés de Graduate Management Admission Test). Este examen se divide en tres secciones, "Verbal", "Quantitative" y "Analytical Writing".

Como puede inferirse, a los aspirantes se les examinan sus habilidades de lectura y comprensión de material escrito, razonamiento cuantitativo y habilidad para la resolución de problemas matemáticos, así como el pensamiento crítico y la capacidad para la expresión de ideas escritas, respectivamente. Nótese que no se les hace ni una sola pregunta sobre el uso de modernas tecnologías, software o internet, tampoco se les pregunta nada sobre las teorías del marketing, contabilidad o finanzas. ¿No le parece extraño?, ¿porqué los aspirantes a estudiar una maestría en negocios en alguna de las universidades más reconocidas del mundo no son cuestionados sobre los temas en los que la educación utilitaria desea poner mayor importancia?

Es claro el objetivo de esta élite de escuelas de negocios, éstas tratan de medir la ca- 
pacidad intelectual de un aspirante enfocándose en lo verdaderamente importante, en las dos piedras angulares de una educación integral, estas son dos de las inteligencias de las que habla el reconocido psicólogo de la Universidad de Harvard, Howard Gardner: 1) la inteligencia lógico-matemática y 2) la inteligencia linguística. Sin duda alguna, el "top ten" de las escuelas de negocios conocen muy bien la importancia de la afirmación de Louis Gerstner Jr., expresidente de IBM, quien ha dicho que "el interés de los dirigentes de empresa no es convertir las escuelas públicas en escuelas vocacionales. Nosotros podemos enseñar a los estudiantes como hacer comercialización. Les podemos enseñar a interpretar balances. Lo que nos resulta muy difícil es tener que enseñar a leer y a calcular, a comunicarse y a pensar". Note usted que las habilidades que demanda el expresidente de IBM son las que se evalúan en el examen GMAT, mismas que deben ser cimentadas desde la formación básica y consolidadas en la educación media superior y superior.

Coincide con esta postura José Antonio Fernández, consultor de la Comunidad Económica Europea, quien afirma que "El rol de la escuela no es producir operarios de lujo, a la medida de las empresas, sino seres humanos pensantes y creativos, que es lo que cada vez más están pidiendo los empresarios más lúcidos y que los políticos, obnubilados con la "tecnología de punta" no tienen en cuenta".

Por último, se debe señalar que durante la aplicación del examen GMAT no se permite el uso de ningún tipo de dispositivo electrónico, calculadora, palm, o mucho menos lap top. Éste es un examen contra el tiempo en donde se apela sólo al uso de lo verdaderamente importante en una persona, su cerebro.

Algo muy parecido ocurre con el examen GRE (Graduate Record Examinations) que es solicitado a los aspirantes a ingresar a reconocidos posgrados en economía e ingeniería. Este examen también se divide en tres áreas que evalúan el razonamiento cuantitativo (Quantitative Reasoning) la comprensión y síntesis de material escrito (Verbal Reasoning) y la habilidad en la expresión escrita (Analytical Writing). Como se puede observar, se ratifica la temática que examina el GMAT, a los candidatos no se les evalúa ningún conocimiento en los que la educación utilitaria desea poner énfasis.

Quienes aspiran a preparar a su hijo para que en un futuro estudie un "Master in Business Administraiton" (MBA) o un Graduate Program in Engineering, en alguna universidad altamente prestigiada, deben procurar que desde la educación básica el niño infiera la importancia superior de las matemáticas y la literatura, logrando así que valore más sus esfuerzos por comprender la cultura asociada a Pitágoras y Aristóteles, que por entender el ambiente amigable que encierran los íconos de Windows.

\section{CULTURA, HUMANISMO Y LITERATURA, ¿PARA QUÉ?}

El criterio utilitario de la educación tiende a excluir la enseñanza de conocimientos que no se les ve -a través de una visión limitada- utilidad práctica o económica. La tendencia utilitaria impulsa a ver con desdén a la educación que fomenta la cultura y el humanismo, sin reflexionar el significado y trascendencia que esto tiene, en gran medida porque no se entiende la utilidad que tienen las humanidades en nuestra vida cotidiana. Ante esta falta de comprensión, es importante comenzar por recordar las palabras de Heidegger al afirmar que "El humanismo consiste en reflexionar y velar porque el hombre sea humano y no in-humano o bárbaro, es decir 
fuera de su esencia”. Entendamos, primero, que las humanidades no son conocimientos destinados a satisfacer aspiraciones de erudición o refinamiento cultural, esta concepción que alcanza sólo los límites de la vanidad personal desvirtúa su razón de ser. El verdadero humanismo debe buscar que sus conocimientos influyan y modelen el comportamiento de una persona, buscan hacerlo más "humano", es decir menos "in-humano".

Para poder entender más esta argumentación es conveniente cuestionarnos sobre algo que afecte nuestra vida cotidiana, como las siguientes dos interrogantes: ¿Alguna vez ha visto usted a algún "bárbaro" al volante de un transporte público?, o si queremos ser más agudos en el sentido de nuestro cuestionamiento, $;$ cree usted que pueda existir algún "bárbaro" dirigiendo alguna "prestigiada empresa" que por el afán económico contamine sin ninguna consideración a nuestro medio ambiente? Las respuestas, que irrefutablemente son afirmativas, nos permiten entender que en ambos casos se trata de personas que están técnicamente capacitadas para realizar su trabajo, uno puede manejar un vehículo y el otro puede dirigir una empresa, pero en ambos casos son personas que se están alejando de un comportamiento marcado por el humanismo, es decir su actuar se aproxima a lo bárbaro o in-humano. Los padres de familia que ponen especial cuidado en que sus hijos aprendan computación o inglés, como lo más importante en su educación, deberían preguntarse si en un futuro les gustaría que sus hijos convivan en una sociedad bilingüe cada vez más in-humana. Bajo esta argumentación es justo recordar una afirmación que hiciera el propio Simón Bolívar, quien advirtió que "El talento sin probidad es un azote".

Por su parte, hace años el Doctor Ignacio Chávez, ex Rector de la UNAM, también explicó que "Ser humanista no significa ser hombre bondadoso [...] ni ilustrado [...] ni cultivar las letras y la historia y el arte, aunque sea útil. Significa, antes que nada, haber adquirido una cultura muy honda que le afine la sensibilidad para ver al hombre con simpatía; haber depurado el juicio para tratar de comprenderlo en sus virtudes y miserias; haber elevado la razón de vida para estar presto a servirlo y ayudarlo en su mejoramiento". Esta es la razón de ser del verdadero humanismo que hoy tanto necesita nuestra sociedad.

En su obra bibliográfica, Jaim Etcheverry recoge una apología al papel que debe tener la cultura en la formación escolar, expuesta por Carlos, Príncipe de Gales, en un aniversario de Shakespeare: "Si decidimos seguir las tendencias de moda en la educación -hacia lo relevante, lo exclusivamente contemporáneo, lo inmediatamente disfrutable- podemos enfrentar el terrible peligro de producir una generación de jóvenes desheredados culturalmente [...] Esto significa no solamente entrenarlos para el trabajo a través de la adquisición de conocimientos, sino, sobre todo, brindarles la posibilidad de comprenderse a sí mismos y el significado profundo de la vida”, y concluía afirmando contundentemente "Resistamos pues, la tentación de negar nuestro legado cultural a tantos jóvenes simplemente por el errado enfoque utilitario. [...] Si bien es loable el énfasis puesto en lo técnico, lo práctico, lo vocacional y lo comercialmente viable, la educación es más que el entrenamiento. Después de todo, carece de sentido llegar a ser técnicamente competentes si, al mismo tiempo, nos volvemos culturalmente ineptos".

Ante la errónea tendencia de la educación utilitaria, los verdaderos educadores deberán, de manera estoica, hacer experimentar en sus educandos los valores que se ganan al practicar la lectura y reflexión de las grandes obras del pensamiento y literatura universal. 
Recientemente, los gurús en el tema de liderazgo empresarial, Warren G. Bennis y James $\mathrm{O}$ 'Toole señalaron que los estudiantes de las escuelas de negocios podrían aprender mucho más sobre liderazgo si tomaran cursos de literatura. Por su parte, Joseph L. Badaracco, profesor de ética de los negocios en Harvard Business School, publicó recientemente un artículo titulado "Atentos a Shakespeare", en el que afirma que "antes que los gurús de moda, los mejores ejemplos sobre liderazgo están presentes en las obras de Sófocles, Shakespeare, Conrad y otros".

Para los que deseen un argumento más pragmático que los antes expuestos, debo mencionar que quienes hemos tenido la oportunidad de impartir clases en alguna escuela de negocios podemos constatar que un alumno con mayor amplitud cultural destaca rápidamente del resto de sus compañeros que sólo se limitan a leer textos técnicos. La inteligencia lingüística se nota inmediatamente y coloca en un nivel intelectualmente superior a quien la ha sabido cultivar desde la infancia.

\section{4. ¿EDUCAR PARA EL ÉXITO PROFESIONAL?}

Es claro percibir que la educación utilitaria tiene como principal orientación educar a las personas para que en un futuro sean capaces de alcanzar un anhelado éxito profesional, entendido éste -para no pocas personascomo una alta posición jerárquica en un trabajo que le permita obtener una importante remuneración económica y disfrutar así de una vida cómoda en una sociedad hedonista, llena de moda, glamour y apariencia.

Se dice que durante una entrevista realizada al famoso Marcel Marceau, en la cual se le preguntaban aspectos sobre su realización personal, éste declaró contundentemente "es que a mí se me educó para ser exitoso, no para ser feliz". La educación que pretende encerrar al hombre en un estrecho círculo de lo que hoy en día se entiende como "éxito profesional" es, en muchos casos, la que estamos fomentando a nuestros educandos. A este tipo de educación me opongo rotundamente. Me permito disentir y buscar otro camino que no sea tan estrecho ni tan superficial. Me uno a la idea de aquellos que tienen la clara conciencia de educar a sus hijos no para que sean hombres exitosos, sí, más bien, para que sean hombres valiosos, es decir, llenos de valores. Apoyo la idea de educar a las personas con sólidos valores humanos para que sean capaces de ver con mayor amplitud y profundidad el sentido de la vida. Me uno a la idea de fomentar una educación integral que dote a las personas de tantos valores humanos, científicos e intelectuales que el éxito profesional les venga por añadidura, pero que sean capaces de controlarlo y encausarlo en una forma de vida digna para un hombre con un nivel más alto de cultura. Como diría el profesor Felix Ovejero "La tarea más importante, la de ser feliz, requiere de un trato inteligente con la vida, buen conocimiento del mundo y de nuestros semejantes para saber orientarnos con destreza y compromiso en el oficio de vivir".

\section{LA CAPACITACIÓN TÉCNICA Y LA ANHELADA EDUCACIÓN CIENTÍFICA}

En nuestro tiempo es muy común escuchar la siguiente afirmación: "El avance tecnológico en los últimos años ha sido enorme". Hay que hacer notar que ante ese avance tecnológico es posible identificar dos clases de sociedades; las que desarrollan la tecnología (sociedad del conocimiento) y aquellas a las que únicamente se les entrena para usarla 
(sociedad dependiente del conocimiento).

A las empresas o países que generan tecnología les conviene que los países en vías de desarrollo se concentren solamente en capacitar a sus sociedades en el uso de la misma. Es cierto que es imposible negar los múltiples beneficios de adoptar tecnologías de punta, sin embargo, tampoco debemos ser ciegos a la dependencia tecnológica que esta práctica produce. Hace años se nos capacito para usar la imprenta, más tarde para el uso de maquinarias textiles y hoy estamos siendo capacitados para usar las más modernas tecnologías de cómputo, sin embargo, en estos tres momentos de nuestra historia el común denominador ha sido el mismo: seguimos siendo entrenados para usar tecnologías ajenas. Entendamos que de seguir así, el verdadero avance tecnológico siempre será de otros, no nuestro.

La sola capacitación técnica nos permite hacer uso de la tecnología, no a comprender el funcionamiento de la misma. Resulta absurdo tratar de ejemplificar esta limitada práctica educativa, es como si alguien llegara a pensar que para formar ingenieros automotrices hay que capacitarlos a través de cursos de manejo en lugar de educarlos con sólidos conocimientos de matemáticas y física aplicada que les permita no sólo aprender a usar el vehículo, sino más bien a desarrollar un vehículo.

Atendiendo a una estrategia educativa de corto o mediano plazo, comparto la necesidad que tiene cualquier país de capacitar técnicamente a diversos sectores de su sociedad para que sean capaces de incrementar su productividad y poder enfrentar el entorno mundialmente competitivo, sin embargo, para el largo plazo esta estrategia resulta completamente insuficiente e incorrecta. Bajo una visión de largo plazo es necesario hacer a un lado el sólo entrenamiento técnico y apos- tarnos, desde la educación básica, por una genuina educación científica que nos enseñe no solo a usar, sino a entender y desarrollar nuestra propia tecnología.

Con base en lo antes argumentado, considero mucho más importante esforzarnos por vincular a nuestros niños y adolescentes con la ciencia a través de profesores que, apasionados de su materia, los acerquen a conferencias en las que descubran el valor de la ciencia, museos interactivos, eficientes programas educativos de ciencia experimental y, a los alumnos más destacados, con los propios institutos de investigación del país, en lugar de adoptar una visión tan corta que pretenda vincularlos con el empleo y el aparato productivo.

La conocida revista Expansión, orientada al sector empresarial en México, entrevistó al Dr. Hector García Molina, miembro del Departamento de Ciencias Informáticas de la Universidad de Stanford, quien se destacó como asesor de las tesis doctorales de los fundadores del conocido sitio web Google. Como parte de la entrevista se le preguntó: "¿Por qué México se encuentra rezagado en tecnología?", la respuesta que este investigador de origen mexicano, quien se desempeña en el más alto nivel de la ciencia y la tecnología en el mundo, debe hacer reflexionar seriamente a los promotores de una educación de corte utilitario al servicio del trabajo. El Doctor García respondió: "El problema no es de capacidad, sino de cultura. En la sociedad mexicana se recompensa a quien se dedica a los negocios [empleo] y no a quien genera conocimiento [ciencia]. En algunos países, ser profesor o tener un doctorado es bien visto. En México, un padre de familia quiere que su hija se case con un abogado o un empresario con mucho dinero. No hay muchos que digan 'Quiero que mi hija se case con un profesor universitario'". Sin ninguna vacilación hay que decir que esta lamenta- 
ble cultura nacional a la que se refiere el Doctor García es la que se fortalecería al acercar a nuestros estudiantes al aparato productivo, ya que se fomentaría cada vez más en ellos la idea del trabajo y de la pronta remuneración económica en lugar fortalecer la cultura de la ciencia y el beneficio a largo plazo.

\section{CONCLUSIONES}

Es absurda esta moda educativa que pretende centrarse en capacitar a las personas a la medida de los requerimientos de las empresas en lugar de apostarnos a educarlas a la altura de las necesidades de nuestros países. Es tanto como aceptar que la visión de país es la visión que tengan las empresas. No es materia de este artículo, sin embargo, no puedo dejar de señalar la incertidumbre y dudas que me causa la reciente moda denominada "educación basada en competencias". ¿No se tratará más bien de una simple capacitación para el trabajo especializado, disfrazada de educación profesional?.

Si algún día se realizara una encuesta con el siguiente cuestionamiento: " Considera usted que la educación debe tener una mayor vinculación con la ciencia y la alta tecnología?", sería muy esperanzador encontrar que las respuestas afirmativas sean tan abrumadoras como las que hoy se observan al tratar de vincular a la educación con empleo.

El sistema educativo básico y medio superior no puede distraer la atención que debe poner en lo fundamental. A nuestros niños y adolescentes hay que vincularlos cada día más al estudio de las matemáticas, las cien- cias, la lengua y la literatura, sólo así se podrá desarrollar en ellos la inteligencia lógico-matemática y lingüística que les permita, en un futuro, aspirar a un desarrollo intelectual más alto y más digno. Sólo así se podrá fortalecer un sistema educativo en favor de la ciencia y la alta tecnología que cualquier país Latinoamericano requiere para ser verdaderamente competitivo. Si sumado a ello, no distraemos la atención que merece el estudio de las humanidades y de las artes, podremos aspirar a que esa ciencia y esa alta tecnología estén siempre bien orientadas, en el rumbo correcto, que es servir y beneficiar a la sociedad.

Concluyo tratando de resumir muchos de los argumentos antes expuestos. Lo hago citando una vez más al ilustre universitario, Dr. Ignacio Chávez, quien, al respecto afirmó "El hombre que sea de hoy y no de ayer, no está ni puede estar contra el avance constante de la tecnología. [...] Apreciado como lo que es, como un medio y no como un fin, el avance técnico es de un valor inestimable [pero sin] caer en la trampa de la tecnificación masiva a costa de nuestra devastación espiritual. Ser amos de las máquinas, no sus esclavos, pidiendo de ellas solo la información, no las decisiones.[...] No interesarse más en el manejo de equipos que en el ejercicio del razonamiento. Metido cada vez más en aparatos $\mathrm{y}$ equipos técnicos $[. .$.$] que rinden mayor \mathrm{y}$ mejor trabajo [una persona irá] poniendo cada día más confianza en sus instrumentos e irá cayendo en un fetichismo lamentable, el fetichismo de los equipos técnicos. Y esto es grave, muy grave [ya que se puede caer] en el culto de la máquina, en vez del [culto al] cerebro". 


\section{REFERENCIAS BIBLIOGRÁFICAS}

1. BADARACCO, Joseph L. "Atentos a Shakespeare", Expansión, Edición especial, Octubre 2006, México, Expansión, pp. 97-104.

2. CHÁVEZ, Ignacio. "Los rápidos avances de la medicina y la lenta, implacable deshumanización de su ejercicio", in Bernardo Sepúlveda (Comp), Humanismo Médico, Educación y Cultura, Tomo I, México, Universidad Nacional Autónoma de México, 1991, pp. 84-94.

3. EDUCATIONAL TESTING SERVICE (ETS), “GRE”, USA, <www.gre.org>, (3 de mayo de 2007).

4. GARDNER, Howard. "La mente ética", Harvard Business Review, Volumen 85 No. 3, Marzo 2007, Chile, Harvard Business School Publishing Corporation, pp. 40-44.
5. GONZÁLEZ, Juliana. El ethos, destino del hombre, México, Universidad Nacional Autónoma de México/Fondo de Cultura Económica, 1997, (1ª. reimpresión), 15 pp.

6. JAIM Etcheverry, Guillermo. La tragedia educativa, Buenos Aires, Fondo de Cultura Económica de Argentina, 2004, (12a . reimpresión), pp. 85-96.

7. MBA.COM, "What Is the GMAT", USA, <http://www.mba.com/mba/TaketheGMAT>, (3 de mayo de 2007).

8. PERALTA, Leonardo. "Una docena de visiones”, Expansión, N. ${ }^{\circ}$ 960, Marzo 2007, México, Expansión, pp. 166-170.

9. SINDICATO NACIONAL DE TRABAJADORES DE LA EDUCACION (SNTE), "Nuestra encuesta", México, <www.snte.org.mx>, (19 de junio de 2007). 